\title{
A MODELLING APPROACH TO EXTRA VIRGIN OLIVE OIL EXTRACTION
}

\author{
Marco Daou, Rocco Furferi, Lucia Recchia, Enrico Cini
}

\section{Introduction}

Current performance of extra virgin olive oil market requires daily updating of quality standards whilst maintaining prices.

Therefore, many specialists have aimed at improving critical working procedures at specific or general levels, as shown in specialized publications $[4,5]$. Recent commercial strategy has shown that requested industrial oil production is capable of obtaining a high quality marketable product. This improved oil production quality can be optimized with automatic controlling systems on the mechanical extraction lines, capable of numerous quality procedural improvements [9].

The improved quality of olive oil extraction can be obtained using specific software able to control the whole process and manage the variables involved. The main objectives of the control process system are minimizing oil quality faults for not perfectly cultivated, picked and stored olives, and maximizing extracted oil quality levels for well cultivated, picked and stored olives.

Study objective has been the realisation of a numeric-modelling instrument for assessing agronomical and technical values, defining olive and process characteristics.

\section{Materials and methods}

\subsection{Model general description}

In order to realize a model capable of assessing operative conditions and process parameters, a specific software has been developed using artificial neural networks $[8,18]$. This software is able to model the

\section{Paper received 31.07.2006; accepted 06.12.2007}

Prof. Eng. Enrico Cini, Dott. Eng. Marco Daou, Ph.D. Eng. LuCIA RECCHIA, Department of Agricultural and Forestry Engineering University of Florence, Italy.

Ph.D. Ing. Rocco FurferI, Department of Mechanics and Industrial Technologies - University of Florence, Italy. extraction process realizing a quantitative and analytical control of extra virgin olive oil quality characteristics $[1,2,3]$. First, using few agronomical parameters (olive harvesting period, ripeness, sanitation, storage and temperature), and few technologic parameters (crusher-mill typology, malaxing-machine typology, temperature and time of malaxing, dilution water percentage, extraction temperature), a model able to evaluate or simulate extracted oil quality has been realized. Particularly, this model allows:

- to obtain prefixed oil quality levels controlling some process parameters during the extraction;

- to simulate a specific extraction process determining, at the same time, olive oil quality.

The process steps considered in this model range from olive sorting to oil extraction. Each step (see Table 1) influences oil quality characteristics (acidity, peroxide number) according to some process parameters and the corresponding influence weight [17] (see Table 2).

Although variable, the weight for the main oil quality values are influenced by agronomical, rather than technical, parameters in olive processing. Oil quality characteristics during olive processing period $[6,7]$ result from geographical and climatic conditions.

\begin{tabular}{|c|c|c|}
\hline & Process phase & Involved parameters \\
\hline \multirow{5}{*}{ 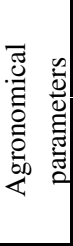 } & \multirow{3}{*}{ Olive harvesting } & Processing period \\
\hline & & Ripeness level \\
\hline & & Sanitation condition \\
\hline & \multirow{2}{*}{ Olive storing } & Storing condition \\
\hline & & Input olive temperature \\
\hline \multirow{6}{*}{ 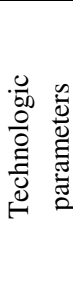 } & Olive milling & Crusher-mill type \\
\hline & \multirow{3}{*}{$\begin{array}{c}\text { Olive paste } \\
\text { malaxing }\end{array}$} & Malaxing-machine type \\
\hline & & Malaxing temperature \\
\hline & & Malaxing time \\
\hline & \multirow{2}{*}{ Oil extraction } & Diluted water percent \\
\hline & & Oil extraction temperature \\
\hline
\end{tabular}

TABLE 1 - Parameters used in modelling process. 


\begin{tabular}{|c|c|}
\cline { 2 - 2 } \multicolumn{1}{c|}{} & phase influence weight \\
\hline Olive harvesting operations & $50 \%$ \\
\hline Olive storing method & $30 \%$ \\
\hline Oil mechanical extraction process & $15 \%$ \\
\hline Oil conservation & $5 \%$ \\
\hline
\end{tabular}

TABLE 2 - Phase influence weight on olive oil quality.

The model realized with its application-oriented software concerns to Tuscany, even though it can be adapted to any other geographical area with similar data to those presented here.

The data used comes from different experimental tests carried out in Tuscany, during olive harvesting from 1997 to 2004 [6, 7, 17]. Using this information, a database of agronomical and technical parameters with corresponding quality-quantity oil extraction values (see Table 1) has been realized. This includes more than 300 samples referred to different olive lots, working periods, extractive processes, geographical areas, etc.

The modelling software has been developed using Matlab ${ }^{\circledR}$ toolboxes; results have been validated by experimental tests held at the "Santa Tea" oil mill in Reggello (Florence) and "Torre Bianca" oil mill in San Casciano in Val di Pesa (Florence); olive oil analysis have been carried out at the Chemical Laboratory - Special Agency of C.C.I.A.A. in Florence.

\subsection{Model development steps}

Work procedure has been developed in the following steps (Fig. 1):

1. creating a homogeneous temporal database, containing all harvest data during the period 1999-2003;

2. creating an artificial neural network system, capable of estimating acidity and peroxide number in extracted oil, malaxing temperature and time, dilution water at decanter inlet.

3. defining acidity and peroxide number estimate with corrective factors that consider other technical parameters in the extractive cycles and agronomical fruit characteristics.

The experimental tests used for this work have been carried out during different years. Moreover, a preventative data homogenization in relation to the database time periods has been necessary: the experimental values of acidity and peroxide number have been multiplied for valid influence factors eliminating processing period influence (see Table 3 ).

Once the homogenized database has been available, a model using artificial neural networks has been implemented, with the aim to define the output values as function of some technical parameters (see Fig. 1). The process period influence factor for each oil quality parameter (acidity and peroxide number) is the ratio between average value made out for a specif- ic process period and the value measured during the reference process period (for example the beginning olive harvesting), considering the annual average amount of experimental samples.

The artificial neural networks $[8,18]$ can be seen as algorithms capable of imitating the irregular learning mechanism of biological organisms. This is a complex system built with calculating cells (neurons), capable of reconstructing the function linking an input to its output, after an iterative training on a correct input-output set. This training ends when the difference (or error) between training output and estimated output is enough small. In this research artificial neural network is used also because it may be easily updated with following collected data.

The calculating system developed consists of FFBP (Feed-Forward Back Propagation) artificial neural networks with sigmoidal activation functions and Levemberg-Marquardt training algorithm [12]. The training set was achieved with 200 samples, each containing 3 inputs and 2 outputs. The inputs are malaxing temperature (tgram) $[293 \mathrm{~K} ; 308 \mathrm{~K}]$, malaxing time (Cgram) [1200 s; $7200 \mathrm{~s}]$, dilution water percentage at decanter inlet $\left(\% \mathrm{H}_{2} \mathrm{O}\right)[0 \% ; 50 \%]$; the outputs are oil peroxide number $(N p)$, oil acidity $(A)$.

This calculating system can be regarded as a vector function $\mathrm{R}^{3} \rightarrow \mathrm{R}^{2}$ : for each input tern (tgram, Cgram, $\left.\% \mathrm{H}_{2} \mathrm{O}\right)$ it computes an output couple $\left(N_{p}, A\right)$. Training completed, oil acidity and oil peroxide number can be calculated comparing new input tern.

The calculating system effectiveness has been checked using database samples not utilized during the training phase.

The comparison between calculated and effective (known) value has shown an average error of $9 \%$. This shift is linked to two parameter evaluation using just three technical parameters, without considering agronomical fruit characteristics and other technical extractive cycle parameters. The achieved outcome is characterized by low accuracy level.

For these reasons, an improving accuracy procedure has been developed, considering other parameters affecting oil quality characteristics $(N p, A)$. Artifi-

\begin{tabular}{|c|l|c|c|}
\hline \multicolumn{2}{|c|}{ PROCESSING PERIOD } & $\begin{array}{c}\text { Oil peroxide } \\
\text { number }(\mathrm{Np}) \\
{\left[\mathrm{meq} \mathrm{O}_{2} / \mathrm{kg}\right]}\end{array}$ & $\begin{array}{c}\text { Oil acidity (A) } \\
{[\% \text { oleic acid] }}\end{array}$ \\
\hline 1 & before November the 6 th & 1,00 & 1,00 \\
\hline 2 & $\begin{array}{l}\text { from November the } 7 \text { th to } \\
\text { the } 16 \text { th }\end{array}$ & 1,30 & 0,93 \\
\hline 3 & $\begin{array}{l}\text { from November the } 17 \text { th } \\
\text { to the } 27 \text { th }\end{array}$ & 1,75 & 0,93 \\
\hline 4 & $\begin{array}{l}\text { from November the } 28 \text { th } \\
\text { to the December 5 }\end{array}$ & 2,50 & 1,00 \\
\hline 5 & $\begin{array}{l}\text { from December the 6th to } \\
\text { the } 14 \text { th }\end{array}$ & 3,50 & 1,12 \\
\hline 6 & After December the 15 th & 4,65 & 1,25 \\
\hline
\end{tabular}

TABLE 3 - Processing period influence factor on oil quality parameters. 


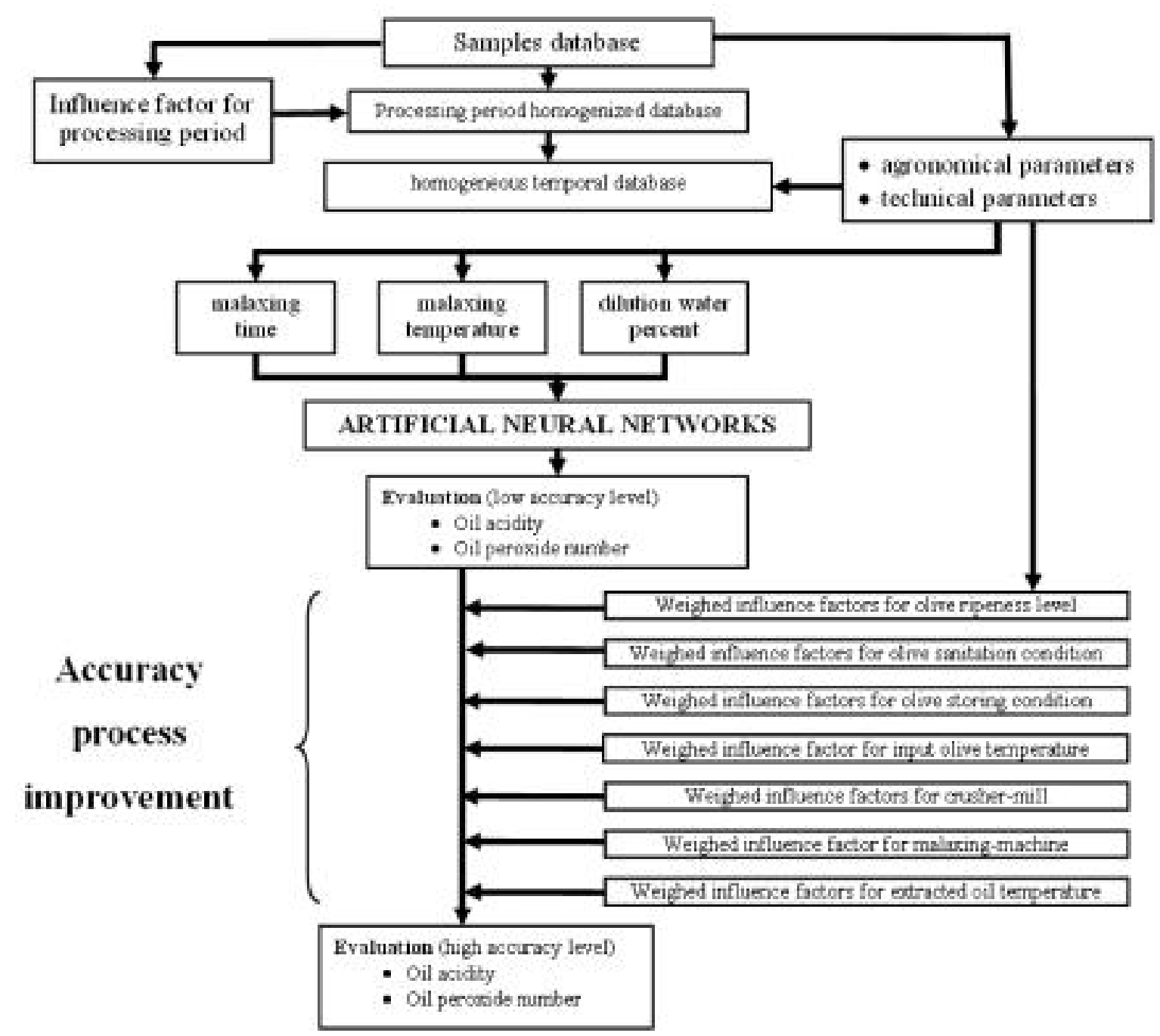

Fig. 1 - Implemented model scheme for olive mill process.

cial neural network output is multiplied by influence factors for agronomical and technical parameters (see Fig. 1). Using experimental database samples, analytical correlations between quality parameters (oil peroxide number and acidity) and both agronomical and technical parameters, not used in neural network systems, are determined. Thus specific influence factors were deterministically obtained: these factors multiply neural network system outputs, increasing system accuracy level.

Working procedure has been defined as follows:

1. oil peroxide number and acidity average values were associated to each process parameter state;

2. population of reliable samples for olive origin, storing and process homogeneity, has been selected for each process parameter;

3. reference-state, characterised by influence factor equal to 1 , has been defined for each process parameter;

4. influence factors of not reference-state have been calculated as ratio between the state average value (oil peroxide number or oil acidity) and the reference-state one;

5. each calculated influence factor, excluding olive processing period, has been calculated using the process phase influence weights. If factor is less than 1 , it is equal to 1 minus the product between two factors: the influence weight concerning the process parameter and the difference between 1 and the process parameter value. For example, the olive storage influence factor equivalent to 0.8 has been corrected to $1-0.3(1-0.8)=0.94$. The difference $(1-0.8)$ depends on storage method $(30 \%)$ and on other parameters $(70 \%)$. If factor is more than 1 , it is equal to 1 plus the product between two factors: the influence weight concerning the process parameter and the difference between the considered process parameter value and 1 . For example, olive storage influence factor equivalent to 1.8 has been corrected to $1+0.3(1.8-1)=1.24$.

\subsection{Influence factor definition}

The weighed influence factor of ripeness for processed olives has been calculated, using different levels of ripeness and considering green olive percentage $(\% V E)$, optimal ripening $(\% I N)$ and advanced ripening $(\% M M)[11]$. The ripeness influence factors 
on oil peroxide number $\left(c \_g m \_N p\right)$ and acidity $\left(c \_g m \_A\right)$ are expressed as follows:

$$
\begin{aligned}
& c \_g m \_N p=0.9 \cdot \frac{\% V E}{100}+1 \cdot \frac{\% I N}{100}+1.105 . \frac{\% M M}{100} \\
& c \_g m \_A=0.79 \cdot \frac{\% V E}{100}+1 \cdot \frac{\% I N}{100}+1.075 . \frac{\% M M}{100}
\end{aligned}
$$

Similarly, the weighed influence factors of sanitation level for oil peroxide number $\left(C \_s s \_N p\right)$ and acidity $\left(c \_s s \_A\right)$, have been defined as weighed average of undamaged olives percentage (\%SA), partially damaged $(\% P D)$, totally damaged $(\% T D)[11]$ :

$$
\begin{aligned}
& c_{-} s s_{-} N p=1 \cdot \frac{\% S A}{100}+0.87 \cdot \frac{\% P D}{100}+1.665 \cdot \frac{\% T D}{100} \\
& c_{-} s s_{-} A=1 \cdot \frac{\% S A}{100}+0.945 \cdot \frac{\% P D}{100}+2.26 \cdot \frac{\% T D}{100}
\end{aligned}
$$

Concerning storage methods $[10,17]$, the influence factors have been obtained using data of sanitation levels, considering a weight of $30 \%$ instead of $50 \%$.

The acidity influence factor (coef_A) of olive temperature at the beginning of the process $\left(T_{\text {olive }}\right)$ has been defined by the following interpolated quadratic equation:

$$
\text { coef_A }=\xi\left(T_{\text {olive }}\right)^{2}+\beta T_{\text {olive }}+\gamma
$$

using $\xi=0,0015, \beta=-0,003, \gamma=0,75$.

Influence factors concerning crusher-mill [7, 17], and malaxing machine $[6,7,11,13,17]$ are shown in Table 4.

The weighed oil temperature influence factors at decanter outlet $\left(T_{\text {ol_us }}\right)$ has been defined as follows:

$$
c \_T_{\text {ol_us }} N p=\left\{\begin{array}{c}
1 \text { per } T_{\text {ol_us }} \leq 302 \mathrm{~K} \\
\delta\left(T_{\text {ol_us }}\right)^{2}-\varepsilon\left(T_{\text {ol_us }}\right)^{2}+\varsigma \\
\text { per } T_{\text {ol_us }} \geq 300 \mathrm{~K}
\end{array}\right.
$$

using $\delta=0,005, \varepsilon=0,3, \zeta=5$;

\begin{tabular}{|c|c|c|c|}
\cline { 3 - 4 } \multicolumn{2}{c|}{} & $\begin{array}{c}\text { Oil peroxide } \\
\text { number }(\mathrm{Np}) \\
{\left[\mathrm{meqO}_{2} / \mathrm{kg}\right]}\end{array}$ & $\begin{array}{c}\text { Oil acidity (A) } \\
{[\% \text { oleic acid] }}\end{array}$ \\
\hline \multirow{4}{*}{$\begin{array}{c}\text { Crusher- } \\
\text { mill }\end{array}$} & $\begin{array}{c}\text { Hisc crushing } \\
\text { crushing }\end{array}$ & 1,000 & 1,000 \\
\cline { 2 - 4 } & Grinding mill & 1.116 & 1.022 \\
\hline \multirow{2}{*}{$\begin{array}{c}\text { Malaxing } \\
\text { machine }\end{array}$} & Horizontal axle & - & 1.071 \\
\cline { 2 - 4 } & Vertical axle & - & 1,0000 \\
\hline
\end{tabular}

TABLE 4 - Crusher-mill and malaxing machine influence factors for oil quality parameters.

$$
c_{-} T_{\text {ol_us }} A=\left\{\begin{array}{c}
1 \text { per } T_{\text {ol_us }} \leq 302 \mathrm{~K} \\
\theta\left(T_{\text {ol_us }}\right)^{2}-\lambda\left(T_{\text {ol_us }}\right)+\mu \\
\text { per } T_{\text {ol_us }} \geq 300 \mathrm{~K}
\end{array}\right.
$$

using $\theta=0,002, \lambda=0,1, \mu=2,3$.

Using data available, nor significant olive temperature at the beginning of the process, neither malaxing machine type influence factors for oil peroxide number have been found.

\subsection{Simulation}

The software developed can be used either for testing extracted oil quality, or for simulating a process and evaluating its performance. Oil temperature at centrifugal decanter outlet cannot be insert without considering other process parameters. The oil temperature must be determined knowing mass and energy balances for process chain (Fig. 2).

The centrifugal decanter can be studied as an open, adiabatic and surface limited system [5, 14, 15], without energetic losses, with two mixed input flows (malaxed paste and dilution water). Using external energy supply, the olive oil paste is centrifugally separated in three output flows: pomace, water with oil traces and oil with water traces (see Table 5). This simplified approach has been developed with the aim to generalize computing procedure; otherwise a detailed model of each machine considering its own technical characteristics must be realized.

\begin{tabular}{|c|c|c|}
\cline { 2 - 3 } \multicolumn{1}{c|}{} & $\begin{array}{c}\text { Density } \\
{\left[\mathrm{kg} / \mathrm{dm}^{3}\right]}\end{array}$ & $\begin{array}{c}\text { Specific heat } \\
{[\mathrm{kJ} / \mathrm{kg} \mathrm{K}]}\end{array}$ \\
\hline Olive oil & 0,92 & 0,10 \\
\hline Water & 1,00 & 0,24 \\
\hline Dry mass & 1,50 & 0,12 \\
\hline
\end{tabular}

TABLE 5 - Density and specific heat of olive compounds.

To simulate an olive oil extraction process, this centrifugal decanter schematization allows to set energy and mass balance equations, from which output decanter parameters can be computed as input functions. Applying this methodology the output temperature of the oil with water traces, its weighed influence factor and olive oil quality can be evaluated (Fig. 2).

The centrifugal decanter energy balance is given as follows:

$$
\begin{gathered}
P t_{e x}+m_{a \_i n} c_{a \_i n} T_{a \_i n}+m_{p a \_i n} c_{p a \_i n} T_{p a \_i n}= \\
=m_{a \_u s} c_{a \_u s} T_{a \_u s}+m_{o l \_u s} c_{o l \_u s} T_{o l \_u s}+m_{s a} c_{s a} T_{s a}
\end{gathered}
$$

where:

$P$ : centrifugal decanter (average) power;

$t_{e x}$ : centrifugal extraction time for whole olive lot;

$m_{a_{-} i n}:$ input dilution water flow;

$c_{a \_ \text {in }}$ : input dilution water specific heat;

$T_{a \_ \text {in }}^{-}$: input dilution water temperature;

$m_{p a \_i n}$ : input malaxed paste flow; 
$c_{p a \text { in }}$ : input malaxed paste specific heat;

$T_{\text {pa } \_ \text {in }}:$ input malaxed paste temperature;

$m_{a \_u s}$ : output water (with oil traces) flow;

$c_{a \_u s}$ : output water (with oil traces) specific heat;

$T_{a \_u s}$ : output water (with oil traces) temperature;

$m_{\text {ol_us }}$ : output oil (with water traces) flow;

$c_{\text {ol_us }}$ : output oil (with water traces) specific heat;

$T_{\text {ol_us }}^{o l u s}$ : output oil (with water traces) temperature;

$m_{s a}:$ output pomace flow;

$c_{s a}$ : output pomace specific heat;

$T_{s a}:$ output pomace temperature.

Parameters shown in (8) have been expressed as function of input software parameters. Using the above balance equation, the oil temperature output can be calculated as fallows:

$$
\begin{aligned}
& T_{o l \_u s}=\frac{1}{m_{o l \_u s} c_{o l \_u s}}\left(P t_{e x}+m_{a \_i n} c_{a \_i n} T_{a \_i n}+\right. \\
& \left.+m_{p a \_i n} c_{p a \_i n} T_{p a \_i n}+m_{a \_u s} c_{a \_u s} T_{a \_u s}+m_{s a} c_{s a} T_{s a}\right)
\end{aligned}
$$

During malaxing phase, each olive cultivar produces paste with specific rheological characteristics. With the aim to simplify the modelling approach, two rheological reference condition have been defined:

PASTE TYPE 1: oil micro-drop coalescence re- quires nor long time, neither high temperature during malaxing process, but water addition can allow easier flow centrifugal separations.

PASTE TYPE 2: rheological characteristics make mechanical processes difficult. To reduce liquid flow emulsions and to improve oil micro-drop coalescences, the increase of malaxing process time and temperature and the reduction of dilution water quantity can allow easier flow centrifugal separation.

Using available data $[11,16]$, the average percentage of mass composition (see Table 6) for each paste type has been determined.

Specific heat of the paste can be calculated for malaxing machine output (or centrifugal decanter input) as weighed average values of mass percentages from Table 6 :

$$
\begin{aligned}
& \mathrm{c}_{1}=0.265 \mathrm{c}_{\mathrm{ol}}+0.415 \mathrm{c}_{\mathrm{aq}}+0.32 \mathrm{c}_{\text {sec }}=0.164 \mathrm{~kJ} / \mathrm{kgK} \\
& \mathrm{c}_{2}=0.225 \mathrm{c}_{\mathrm{ol}}+0.485 \mathrm{c}_{\mathrm{aq}}+0.29 \mathrm{vc}_{\text {sec }}=0.173 \mathrm{~kJ} / \mathrm{kgK}
\end{aligned}
$$

where:

$c_{1}$ : type 1 paste specific heat;

$c_{2}$ : type 2 paste specific heat;

$c_{o l}$ : oil specific heat;

$c_{a q}$ : water specific heat;

$c_{s e c}$ : dry mass specific heat.

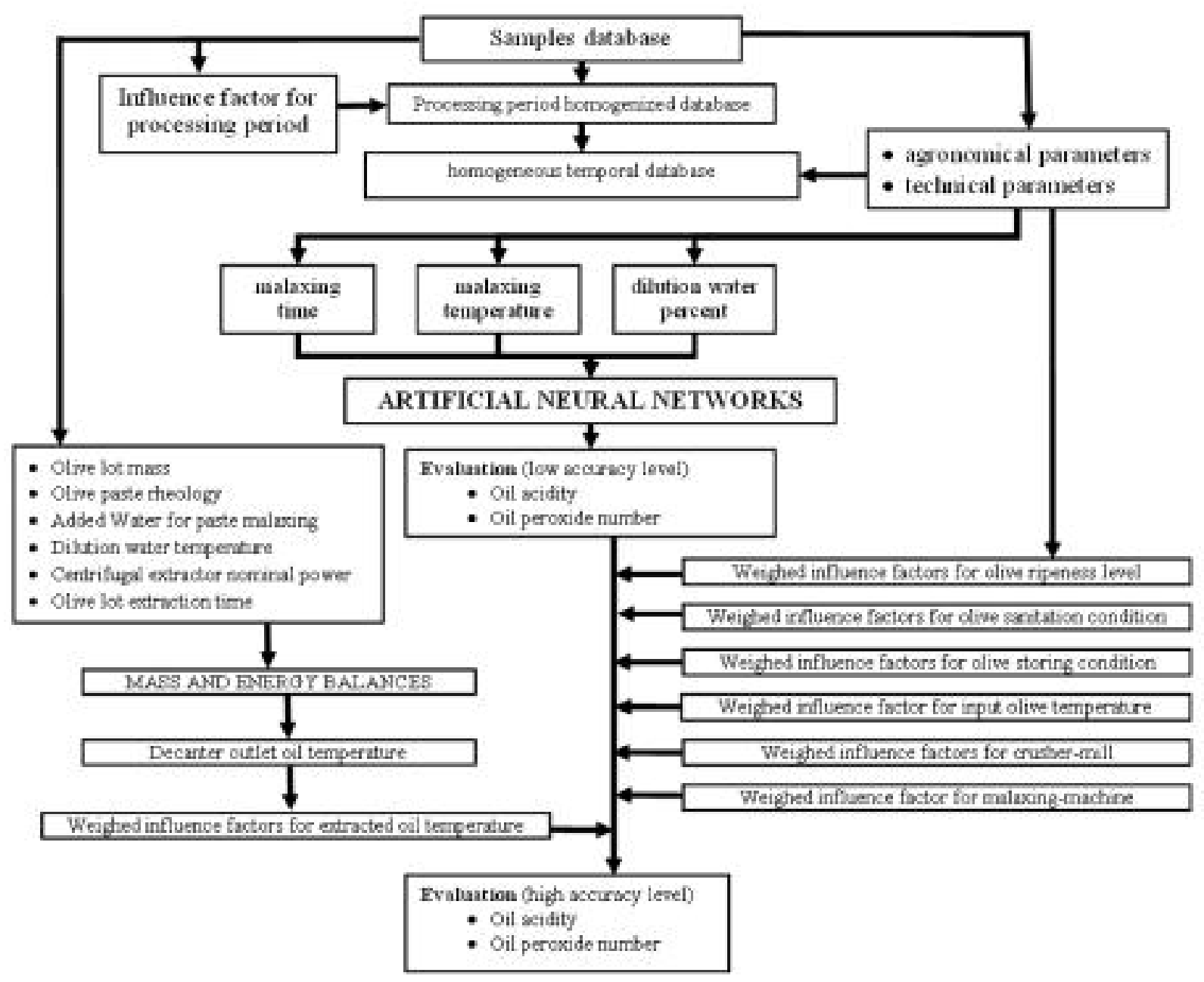

Fig. 2 - Software simulating mode running. 


\begin{tabular}{|c|c|c|c|}
\cline { 2 - 4 } \multicolumn{1}{c|}{} & $\%$ oil & $\%$ water & $\%$ dry mass \\
\hline Type 1 paste & 26,5 & 41,5 & 32,0 \\
\hline Type 2 paste & 22,5 & 48,5 & 29,0 \\
\hline
\end{tabular}

TABLE 6 - Example of malaxed paste mass composition.

Similarly, specific heat can be calculated for centrifugal decanter outlet flows (water containing oil traces, oil containing water traces, pomace) as follows:

$$
\begin{aligned}
& \mathrm{c}_{s a}=0.035 \mathrm{c}_{o l}+0.365 \mathrm{c}_{a q}+0.60 \mathrm{c}_{s e c}=0.162 \mathrm{~kJ} / \mathrm{kgK} \\
& \mathrm{c}_{a \_u s}=0.01 \mathrm{c}_{o l}+0.90 \mathrm{c}_{a q}+0.09 \mathrm{c}_{\text {sec }}=0.227 \mathrm{~kJ} / \mathrm{kgK} \\
& \mathrm{c}_{\text {ol_us }}=0.90 \mathrm{c}_{o l}+0.09 \mathrm{c}_{a q}+0.01 \mathrm{c}_{\text {sec }}=0.112 \mathrm{~kJ} / \mathrm{kgK}
\end{aligned}
$$

Available data regarding outlet flows from centrifugal decanter [6] have shown difference between water temperature (containing oil traces) and oil temperature (containing water traces), varying in the range $[0.5 ; 2.2] \mathrm{K}$ with an average value of $1,4 \mathrm{~K}$ :

$$
T_{a \_u s}=T_{o l \_u s}-1.4
$$

Three-flow decanter data available report pomace temperature lower $(-1 \mathrm{~K})$ than water temperature, as following from centrifugal decanter model: centrifuged mass internal friction transforms kinetic centrifugal energy into heat; heat is conducted from decanter shaft towards rotational drum, causing temperature increasing according to each compound specific heat. Water, having an high specific heat and interposing between oil and pomace, determines an higher water-pomace temperature gradient than oil-water one:

$$
T_{s a}=T_{a \_u s}-1
$$

A three equation system has been drawn for the decanter inlet and outlet mass balance, considering the whole extraction time $\left(t_{\text {estr }}\right)$ :

$$
\begin{aligned}
& m_{\text {ol_in }}=0.01 m_{a \_u s}+0.9 m_{\text {ol_us }}+0.035 m_{s a} \\
& m_{a \_ \text {in }}=0.9 m_{a \_u s}+0.09 m_{\text {ol_us }}+0.365 m_{s a} \\
& m_{s e c}=0.09 m^{-}+0.01 m+0.6 m^{-u s}
\end{aligned}
$$

The system unknowns are centrifugal decanter outlet flows $m_{a \_u s}, m_{o l \_u s}, m_{s a}$, while $m_{o l \text { in }}, m_{a_{i} \text { in }}, m_{\text {sec }}$ are known process parameters expressed as functions of olive lot mass $\left(m_{\text {olive }}\right)$, dilution water percentage at decanter inlet related to olive mass (\% H2O), additional water percentage at malaxing machine $(\alpha)$ and paste type:

\section{TYPE 1 PASTE}

$$
\left\{\begin{array}{l}
0.265 m_{\text {olive }}=0.01 m_{a \_u s}+0.9 m_{\text {ol_us }}+0.035 m_{s a} \\
\left(0.415+\% H_{2} O+\alpha\right) m_{\text {olve }}= \\
=0.9 m_{a \_u s}+0.9 m_{\text {ol_us }}+0.365 m_{s a} \\
0.32 m_{\text {olive }}=0.09 m_{a_{-} u s}+0.01 m_{\text {ol_us }}+0.6 m_{s a}
\end{array}\right.
$$

\section{TYPE 2 PASTE}

$$
\left\{\begin{array}{l}
0.225 m_{\text {olive }}=0.01 m_{a \_u s}+0.9 m_{\text {ol_us }}+0.035 m_{s a} \\
\left(0.485+\% H_{2} O+\alpha\right) m_{\text {olive }}= \\
=0.9 m_{a \_u s}+0.09 m_{\text {ol_us }}+0.365 m_{\text {sa }} \\
0.29 m_{\text {olive }}=0.09 m_{\text {a_us }}+0.01 m_{\text {ol_us }}+0.6 m_{\text {sa }}
\end{array}\right.
$$

The system solutions are centrifugal decanter outlet mass flows as function of olive lot mass:

\section{TYPE 1 PASTE}

$$
\begin{aligned}
m_{\text {a_us }}=\left[0.236+1.183\left(\% \mathrm{H}_{2} \mathrm{O}+\alpha\right)\right] m_{\text {olive }} \\
m_{\text {ol_us }}=\left[0.273-0.00629\left(\% \mathrm{H}_{2} \mathrm{O}+\alpha\right)\right] m_{\text {olive }} \\
m_{\text {sa }}=\left[0.490-0.176\left(\% \mathrm{H}_{2} \mathrm{O}+\alpha\right)\right] m_{\text {olive }} \\
\operatorname{TYPE~2~PASTE~} \\
m_{\text {a_us }}=\left[0.337+1.183\left(\% \mathrm{H}_{2} \mathrm{O}+\alpha\right)\right] m_{\text {olive }} \\
m_{\text {ol_us }}=\left[0.229-0.00629\left(\% \mathrm{H}_{2} \mathrm{O}+\alpha\right)\right] m_{\text {olive }} \\
m_{\text {sa }}=\left[0.439-0.176\left(\% \mathrm{H}_{2} \mathrm{O}+\alpha\right)\right] m_{\text {olive }}
\end{aligned}
$$

where:

$\% \mathrm{H}_{2} \mathrm{O} \mathrm{m}_{\text {olive }}$ indicates dilution water percentage at centrifugal decanter inlet normalizing by olive mass;

$\alpha m_{\text {olive }}$ indicates additional water in malaxed paste normalizing by olive mass.

Oil temperature $\left(T_{\text {ol } \_s}\right)$ has been calculated from mass flow and temperature expressions related to decanter outputs:

\section{TYPE 1 PASTE}

$$
T_{o l \_u s}=\frac{J+(1+\alpha) 0.686 T_{p a \_i n}+1.113+1.286 K}{0.685+K}
$$

TYPE 2 PASTE

$$
T_{o l \_u s}=\frac{J+(1+\alpha) 0.725 T_{p a \_i n}+1.166+1.286 K}{0.727+K}
$$

where:

$$
K=\left(\% \mathrm{H}_{2} \mathrm{O}+\alpha\right) \quad J=\% \mathrm{H}_{2} O \cdot T_{\text {aq_ing }}+\frac{P \cdot t_{\text {ex }}}{m_{\text {olive }}}
$$

Using simulation model, decanter inlet paste temperature $\left(T_{p a i n}\right)$ has been set at approximately average malaxing temperature using software in simulating mode.

Using weighed averages and calculated mass flows, the software can be applied to two-flow or three-flow decanters.

\section{Results}

Exceptional climatic conditions verified in Tuscany during the 2003 decrease olive production of 60$80 \%$, even $90 \%$ in some areas. Therefore initial model testing was very difficult because of production area and oil-mill identifications. To overcome these difficulties, software validation procedure has taken a long time and was divided into two distinct steps: verification and validation. The first step (verification) verifies that software outputs present a sufficient accuracy 
level in relation to oil peroxide number and acidity values of the samples not used during software training. The second step (validation), has been conducted using 2003 and 2006 olive oil samples. The 2003 sample (Table 7) was taken from a three-flow de-

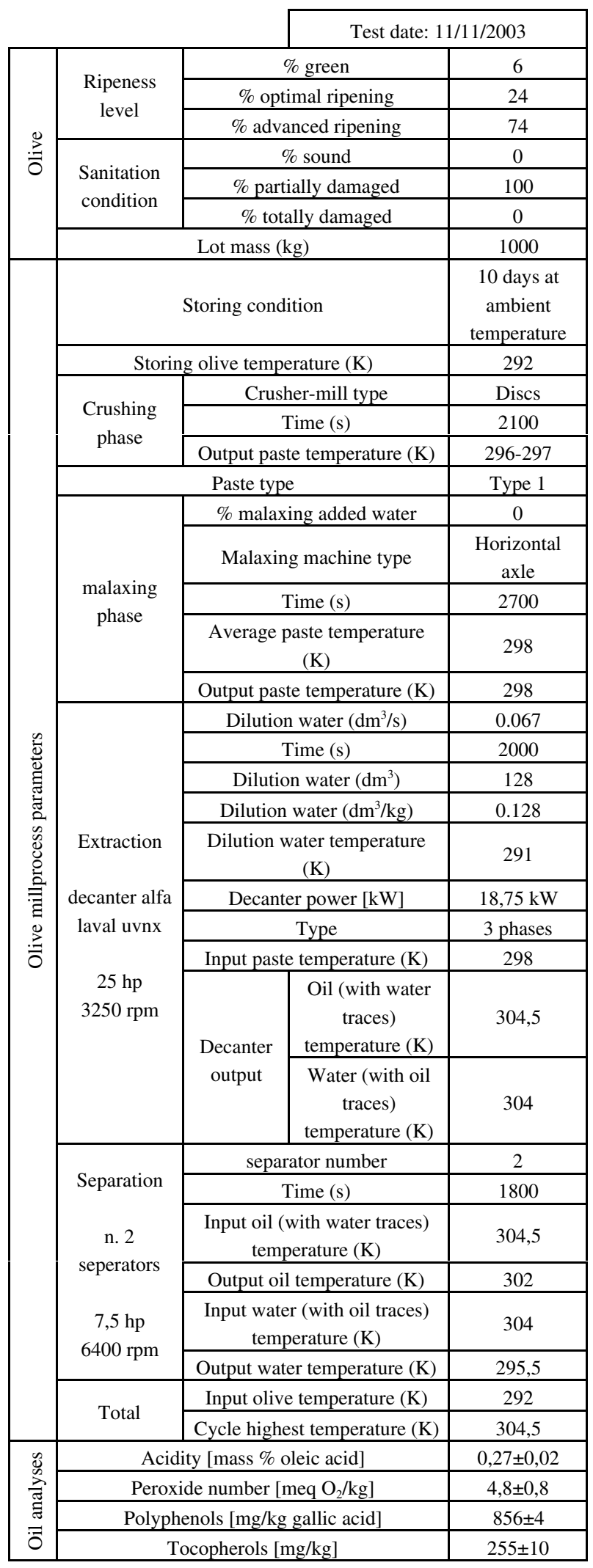

TABLE 7 - Performed experiment for software testing. canter oil mill with two low temperature final separators ("Santa Tea" oil mill in Reggello (Florence)); 2006 samples were taken from a two-flow decanter oil mill with without final separators ("Torre Bianca" oil mill in San Casciano in Val di Pesa (Florence)).

Tables 8 and 9 compare database samples with software outputs concerning verification and validation phases as described above. It appears how soft-

\begin{tabular}{|c|c|c|c|c|}
\cline { 2 - 5 } \multicolumn{1}{c|}{} & \multicolumn{2}{c|}{$\begin{array}{c}\text { Peroxide number (Np) } \\
\text { [meq } \mathrm{O}_{2} / \mathrm{kg} \text { ] }\end{array}$} & \multicolumn{2}{c|}{$\begin{array}{c}\text { Acidity (A) } \\
\text { [mass \% oleic acid] }\end{array}$} \\
\hline $\begin{array}{c}\text { Sample } \\
\text { label }\end{array}$ & $\begin{array}{c}\text { Analyses } \\
\text { results [6] }\end{array}$ & $\begin{array}{c}\text { Software } \\
\text { output }\end{array}$ & $\begin{array}{c}\text { Analyses } \\
\text { results [6] }\end{array}$ & $\begin{array}{c}\text { Software } \\
\text { output }\end{array}$ \\
\hline 12_00f04o1 & $6 \pm 2$ & 6 & $0,18 \pm 0,01$ & 0,18 \\
\hline 12_00f04v1 & $7 \pm 2$ & 7,5 & $0,21 \pm 0,01$ & 0,22 \\
\hline 12_00f04v2 & $6 \pm 2$ & 6,2 & $0,23 \pm 0,01$ & 0,24 \\
\hline 12_00f10o1 & $6 \pm 2$ & 5,4 & $0,17 \pm 0,01$ & 0,16 \\
\hline 12_00f10v1 & $7 \pm 2$ & 5,7 & $0,19 \pm 0,01$ & 0,2 \\
\hline 12_00f10v2 & $6 \pm 2$ & 6,8 & $0,21 \pm 0,01$ & 0,23 \\
\hline 12_00f17o1 & $5 \pm 2$ & 6,4 & $0,17 \pm 0,01$ & 0,19 \\
\hline 12_00f17v1 & $5 \pm 2$ & 6,6 & $0,21 \pm 0,01$ & 0,27 \\
\hline 12_00f17v2 & $5 \pm 2$ & 6,2 & $0,22 \pm, 0,01$ & 0,26 \\
\hline 12_00f24o1 & $6 \pm 2$ & 6,4 & $0,22 \pm, 0,01$ & 0,21 \\
\hline 12_00f24v1 & $6 \pm 2$ & 6,2 & $0,28 \pm, 0,01$ & 0,27 \\
\hline 12_00f24v2 & $5 \pm 2$ & 5,9 & $0,23 \pm, 0,01$ & 0,24 \\
\hline 12_01f03o1 & $4 \pm 2$ & 3,6 & $0,17 \pm 0,01$ & 0,15 \\
\hline 12_01f17o1 & $5 \pm 2$ & 5,1 & $0,14 \pm 0,01$ & 0,11 \\
\hline 12_01f17v1 & $8 \pm 2$ & 7 & $0,13 \pm 0,01$ & 0,16 \\
\hline 12_01f24o1 & $2 \pm 2$ & 1,9 & $0,20 \pm 0,01$ & 0,15 \\
\hline 12_02f09o1 & $4 \pm 2$ & 4 & $0,18 \pm 0,01$ & 0,18 \\
\hline 12_02f09v1 & $4 \pm 2$ & 5,1 & $0,25 \pm 0,01$ & 0,25 \\
\hline 12_02f20o1 & $4 \pm 2$ & 4,3 & $0,15 \pm 0,01$ & 0,19 \\
\hline 12_02f20v1 & $4 \pm 2$ & 4,8 & $0,28 \pm 0,01$ & 0,26 \\
\hline AVERAGE & 5,6 & 5,2 & 0,20 & 0,21 \\
\hline
\end{tabular}

TABLE 8 - Comparison between database samples and software outputs (verification).

\begin{tabular}{|c|c|c|c|c|}
\cline { 2 - 5 } \multicolumn{1}{c|}{} & \multicolumn{2}{c|}{$\begin{array}{c}\text { Peroxide number (Np) } \\
\left.\text { [meq } \mathrm{O}_{2} / \mathrm{kg}\right]\end{array}$} & \multicolumn{2}{c|}{$\begin{array}{c}\text { Acidity (A) } \\
\text { [mass \% oleic acid] }\end{array}$} \\
\hline Sample label & $\begin{array}{c}\text { Analyses } \\
\text { results [6] }\end{array}$ & $\begin{array}{c}\text { Software } \\
\text { output }\end{array}$ & $\begin{array}{c}\text { Analyses } \\
\text { results [6] }\end{array}$ & $\begin{array}{c}\text { Software } \\
\text { output }\end{array}$ \\
\hline 2003_ST_01 & $4,8 \pm 0,8$ & $0,27 \pm 0,02$ & 4,8 & 0,26 \\
\hline 2006_TB_09 & $4,8 \pm 0,8$ & $0,21 \pm 0,01$ & 3,7 & 0,16 \\
\hline 2006_TB_12 & $4,3 \pm 0,8$ & $0,16 \pm 0,01$ & 3,9 & 0,19 \\
\hline 2006_TB_20 & $3,9 \pm 0,8$ & $0,23 \pm 0,01$ & 4,1 & 0,21 \\
\hline 2006_TB_21 & $3,6 \pm 0,8$ & $0,18 \pm 0,01$ & 3,9 & 0,18 \\
\hline 2006_TB_22/1 & $3,3 \pm 0,8$ & $0,18 \pm 0,01$ & 3,7 & 0,16 \\
\hline 2006_TB_22/2 & $4,3 \pm 0,8$ & $0,17 \pm 0,01$ & 3,7 & 0,16 \\
\hline 2006_TB_25 & $3,9 \pm 0,8$ & $0,20 \pm 0,01$ & 3,9 & 0,17 \\
\hline 2006_TB_40 & $3,8 \pm 0,8$ & $0,13 \pm 0,01$ & 3,9 & 0,18 \\
\hline 2006_TB_50 & $3,9 \pm 0,8$ & $0,16 \pm 0,01$ & 5,1 & 0,18 \\
\hline 2006_TB_60 & $6,7 \pm 0,8$ & $0,17 \pm 0,01$ & 5,0 & 0,16 \\
\hline 2006_TB_70 & $3,8 \pm 0,8$ & $0,14 \pm 0,01$ & 5,0 & 0,17 \\
\hline 2006_TB_75 & $5,5 \pm 0,8$ & $0,16 \pm 0,01$ & 4,9 & 0,16 \\
\hline AVERAGE & 4,4 & 0,18 & 4,3 & 0,18 \\
\hline
\end{tabular}

TABLE 9 - Comparison between validation samples (chemical analyses) and software outputs. 


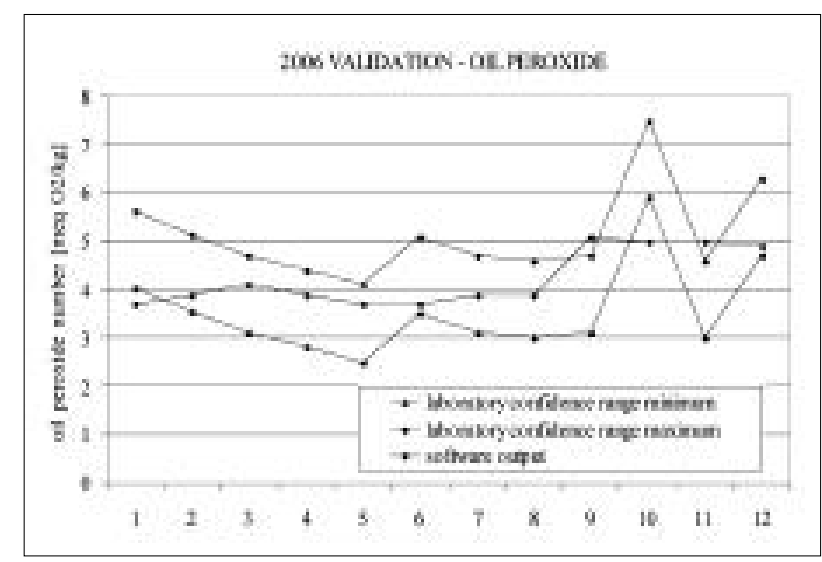

Fig. 3 - Comparison of oil peroxide number between software outputs and 2006 samples (validation).

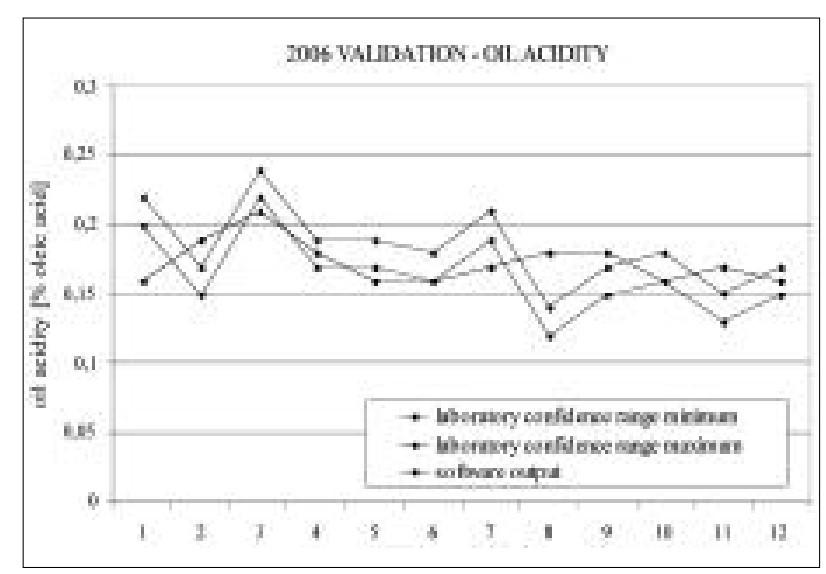

Fig. 4 - Comparison of oil acidity between software outputs and 2006 samples (validation).

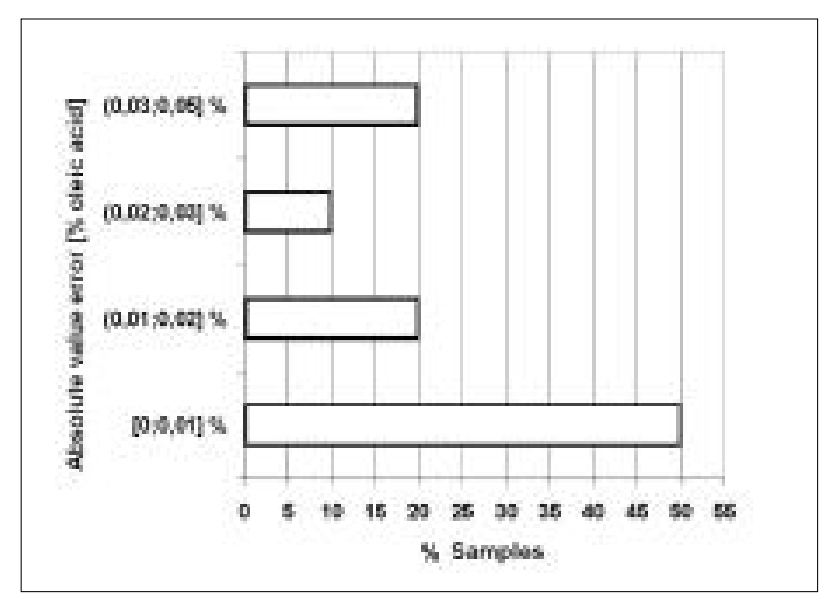

Fig. 5 - Software oil acidity estimation error distribution (verification).

ware output is consistent as shown by average value either in verification and in validation process. Software determines oil peroxide number value into laboratory confidence range for each sample, as shown by chemical analyses results (see Fig. 3).

Concerning acidity output, software has performed well: compared to laboratory confidence range central value, software output shows an error distribution confirming approach effectiveness (see Fig. 4, 5).

\section{Conclusions}

Comparing laboratory and software results, this initial software development phase can be deemed valid (see Table 9). However, further improvements are required. This modelling software can be used in automatic control mode or in simulating mode and it can reduce laboratory analyses number, reducing laboratory analyses number.

Further specific tests could quantify the influence of various parameters concerning olive oil quality (cultivation, olive tree micro-environment, storing conditions, paste granulometry, etc.). These added information could extend software coverage to the whole olive oil productive line.

This research shows the feasibility of an algorithmic modelling approach to olive oil production, using deterministic or stochastic tools according to numerousness of available data. The availability of wide sample set could make it possible to apply the developed methodology to specific olive mill.

\section{Acknowledgments}

Some data used in this work have been taken from experimental database of Laboratorio Chimico e Merceologico della Camera di Commercio in Firenze. Authors would like to thank Marzia Migliorini, Marco Mugelli, Paolo Viti, Massimo Pasquini for data supply.

\section{References}

[1] A.A.V.v., Regolamento CEE $n^{\circ}$ 2568/91, G.U. CEE n. L 248 del 05/09/91 e successive modificazioni

[2] A.A.V.v., Regolamento CEE $n^{\circ}$ 2081/92, G.U. CEE n. L 208 del 24/07/92 e successive modificazioni

[3] A.A.Vv., Regolamento (CE) n. 796/2002, G.U. CE n. L 128/8 del 15.5.2002.

[4] Amirante P., Di Rienzo G.C., Di Giovacchino L., Bianchi B., Catalano P., Evoluzione tecnologica degli impianti di estrazione dell'olio di oliva. OLIVÆ (1993) 48, 43-53.

[5] Amirante P., Catalano P., Analisi teorica e sperimentale dell'estrazione dell'olio d'oliva per centrifugazione, Rivista Italiana delle Sostanze Grasse, Volume LXX, Luglio (1993) 329-335.

[6] Amirante P., Cini E., Montel G.L., Pasqualone A., Tecnologie innovative per l'estrazione dell'olio vergine di oliva in Toscana: influenza dei parametri di processo sulla qualità, Estratto da "L'innovazione tecnologica per l'agricoltura di precisione e la qualità produttiva”, Grugliasco - 22/23 Giugno 1999.

[7] Amirante P., Pasqualone A., Montel G.L., Catalano P., Cini E., Di Giovacchino L., Clodoveo M.L., BACCIONI L., Cresti G., Influenza delle tec- 
niche estrattive sulla qualità dell'olio in Toscana, Nuovi sistemi di coltivazione dell'olivo, Atti ARSIA (2001) 63-67.

[8] BIshop C.M., Neural Networks for Pattern Recognition, Oxford University Press Inc., New York (1995).

[9] Catalano P., Cini E., Sarghini F., Applicazione di modelli nell'agroindustria per la ricerca e la gestione dei sistemi produttivi. Atti del Convegno "Innovazione delle macchine e degli impianti nel settore agroalimentare per un' agricoltura multifunzionale nel rispetto dell'ambiente", Anacapri 5-6 Giugno 2006.

[10] ÇAVAsoglu A., OKTAR A., Gli effetti dei fattori agronomici e delle condizioni di stoccaggio prima della frangitura sulle qualità dell'olio d'oliva. OLIVÆ (1994) 52, 18-24.

[11] Cini E., Magni J., Migliorini M., Mugelli M., Pasquini M., Recchia L., Sperimentazione di una gramola ad asse verticale nell'estrazione di olio di oliva. Riv. Ing. Agr. (2006) 1, 1-10.

[12] Davis P., Levenberg-Marquart Methods and Nonlinear Estimation, SIAM News (1993) Vol. 26, Number 6.

[13] Di Giovacchino L., L'estrazione dell'olio con la centrifugazione diretta della paste di olive. Nota I: Influenza della gramolazione. Rivista Italiana delle Sostanze Grasse, Volume LXVIII, Agosto 1991, 413420.

[14] Di Giovacchino L., Mucciarella M.R., Costantini N., FERRANTE M.L., I decanter centrifughi innovativi con recupero d'acqua. Influenza della portata di alimentazione delle paste di olive sulle rese e sulla qualità dell'olio. Rivista Italiana delle Sostanze Grasse, (1997) 9, 405-408.

[15] Di Giovacchino L., Mascolo A., Incidenza delle tecniche operative nell'estrazione dell'olio dalle olive con il sistema continuo. Nota II. Rivista Italiana delle Sostanze Grasse (1998) 4, 283-289.

[16] Lanzani A., Bondioli P., Mariani C., Folegatti L., FEDELI E., Influenza dei parametri tecnologici sulla qualità degli oli vergini di oliva nella pratica industriale. Nota II. Rivista Italiana delle Sostanze Grasse, Volume LXX, Maggio 1993, 223-232.

[17] Mugelli M., Cini E., Migliorini M., Simeani G., Modi G., Migliarese F., Sistemi di frangitura: influenza sulle caratteristiche chimiche ed organolettiche dell'olio di oliva extravergine. Ed. Camera di Commercio I.A.A. di Firenze Laboratorio Chimico Merceologico - Azienda speciale della C.C.I.A.A. di Firenze, Litografia I.P., 1998.

[18] RIPLey B.D., Pattern Recognition and Neural Network. Cambridge University Press, Cambridge, 1996.

\section{SUMMARY}

In the present work is described a feasibility assessment for a new approach in virgin olive oil production control system. A predicting or simulating algorithm is implemented as artificial neural network based software, using literature found data concerning parameters related to olive grove, process, machine. Test and validation proved this tool is able to answer two different frequently asked questions by olive oil mill operators, using few agronomic and technological parameters with time and cost saving:
- which quality level is up to oil extracted from defined olive lot following a defined process (predicting mode);

- which process and machine parameters set would determine highest quality level for oil extracted from a defined olive lot (simulating mode).

\section{Key words:}

Olive oil, Control system, Prediction algorithm.

\section{Symbols}

$\%$ IN percentage of medium ripening olives in the lot [dimensionless];

$\% M M$ percentage of advanced ripening $m_{\text {olive }}$ olives in the lot [dimensionless];

$\% P D$ percentage of partially damaged olives in the lot [dimensionless];

$\% S A$ percentage of intact olives in the lot [dimensionless];

$\% T D$ percentage of totally damaged olives in the lot [dimensionless];

$\% V E$ percentage of green olives in the lot [dimensionless];

$\% H 20$ decanter dilution water percent;

$A \quad$ oil acidity (mass percentage of oleic acid) [dimensionless];

$c \_g m \_N p$ influence factor of olive ripening on oil peroxide number [dimensionless];

$c \_g m \_A$ influence factor of olive ripening on oil acidity [dimensionless];

$c \_s s \_N p$ influence factor of olive ripening on oil peroxide number [dimensionless];

$c \_s s \_A$ influence factor of olive ripening on oil acidity [dimensionless];

$c \_T_{o l \_u s} A$ maximum oil temperature factor on oil acidity [dimensionless];

$c_{-} T_{\text {ol } u s \_} N p$ maximum oil temperature factor on oil peroxide number [dimensionless];

$c_{1} \quad$ type 1 malaxed paste specific heat $[\mathrm{kJ} / \mathrm{kgK}]$;

$c_{2} \quad$ type 2 malaxed paste specific heat $[\mathrm{kJ} / \mathrm{kgK}]$;

$c_{a \_ \text {in }}$ decanter input dilution water specific heat $[\mathrm{kJ} / \mathrm{kgK}]$;

$c_{a \_u s} \quad$ decanter output water (with oil traces) specific heat $[\mathrm{kJ} / \mathrm{kgK}]$;

$c_{o l} \quad$ oil specific heat $[\mathrm{kJ} / \mathrm{kgK}]$;

$c_{a q} \quad$ water specific heat $[\mathrm{kJ} / \mathrm{kgK}]$;

$c_{\text {sec }} \quad$ olive dry mass specific heat $[\mathrm{kJ} / \mathrm{kgK}]$;

$c_{\text {ol_us }}$ decanter output oil (with water traces) specific heat $[\mathrm{kJ} / \mathrm{kgK}]$;

$c_{s a}$ decanter output pomace specific heat $[\mathrm{kJ} /$ $\mathrm{kgK}]$;

$c_{\text {pa_in }}$ decanter input malaxed paste specific heat $[\mathrm{kJ} / \mathrm{kgK}]$;

Cgram average malaxing temperature of olive paste $[\mathrm{K}]$;

coef_A starting process olive temperature influence factor on oil acidity $[\mathrm{K}]$;

$m_{a \_ \text {in }} \quad$ decanter input dilution water flow $[\mathrm{kg} / \mathrm{s}]$; 
$m_{a u s}$ decanter output water (with oil traces) flow $[\mathrm{kg} / \mathrm{s}]$;

$m_{\text {ol_in }} \quad$ decanter input oil flow $[\mathrm{kg} / \mathrm{s}]$;

$m_{\text {ol_us }}$ decanter output oil (with water traces) flow $[\mathrm{kg} / \mathrm{s}]$;

$m_{\text {olive }} \quad$ olive lot mass $[\mathrm{kg}]$;

$m_{p a \_ \text {in }}$ decanter input malaxed paste flow $[\mathrm{kg} / \mathrm{s}]$;

$m_{s a} \quad$ decanter output pomace flow $[\mathrm{kg} / \mathrm{s}]$;

$m_{\mathrm{sec}} \quad$ decanter input dry mass flow è $\left.\mathrm{kg} / \mathrm{s}\right]$;

$N_{p} \quad$ oil number of peroxide $\left[\mathrm{m}_{\mathrm{eqO} 2} / \mathrm{kg}\right]$

$P^{p} \quad$ decanter (average) power $[\mathrm{kW}]$;

tgram malaxing time of olive paste $[\mathrm{s}]$;

$T_{a \_ \text {in }} \quad$ decanter input dilution water temperature $[\mathrm{K}]$;
$T_{a \_ \text {s }}$ decanter output water (with oil traces) temperature $[\mathrm{K}]$;

$T_{\text {ol_us }}$ decanter output oil (with water traces) temperature $[\mathrm{K}]$;

$T_{s a} \quad$ decanter output pomace temperature $[\mathrm{K}]$;

$T_{\text {ol_us }}$ maximum oil temperature during extraction $[\mathrm{K}]$;

$T_{\text {olive }} \quad$ starting process olive temperature $[\mathrm{K}]$;

$T_{\text {pa in }}$ decanter input malaxed paste temperature $[\mathrm{K}]$;

$t_{e x} \quad$ olive lot extraction time [s]. 